\title{
Matrix methods for perfect signal recovery underlying range space of operators
}

\author{
Fahimeh Arabyani Neyshaburi ${ }^{1}$, Ramin Farshchian ${ }^{1}$, and Rajab Ali Kamyabi-Gol ${ }^{1}$ \\ ${ }^{1}$ Ferdowsi University of Mashhad
}

December 10, 2021

\begin{abstract}
The purpose of this work is to investigate perfect reconstruction underlying range space of operators in finite dimensional Hilbert spaces by a new matrix method. To this end, first we obtain more structures of the canonical $\$ \mathrm{~K} \$$-dual. \% and survey optimal $\$ \mathrm{~K} \$$-dual problem under probabilistic erasures. Then, we survey the problem of recovering and robustness of signals when the erasure set satisfies the minimal redundancy condition or the $\$ \mathrm{~K} \$$-frame is maximal robust. Furthermore, we show that the error rate is reduced under erasures if the $\$ \mathrm{~K} \$$-frame is of uniform excess. Toward the protection of encoding frame (K-dual) against erasures, we introduce a new concept so called $\$(\mathrm{r}, \mathrm{k}) \$$-matrix to recover lost data and solve the perfect recovery problem via matrix equations. Moreover, we discuss the existence of such matrices by using minimal redundancy condition on decoding frames for operators. We exhibit several examples that illustrate the advantage of using the new matrix method with respect to the previous approaches in existence construction. And finally, we provide the numerical results to confirm the main results in the case noise-free and test sensitivity of the method with respect to noise.
\end{abstract}

\section{Hosted file}

Manuscript .pdf available at https://authorea.com/users/450605/articles/548899-matrix-methodsfor-perfect-signal-recovery-underlying-range-space-of-operators 